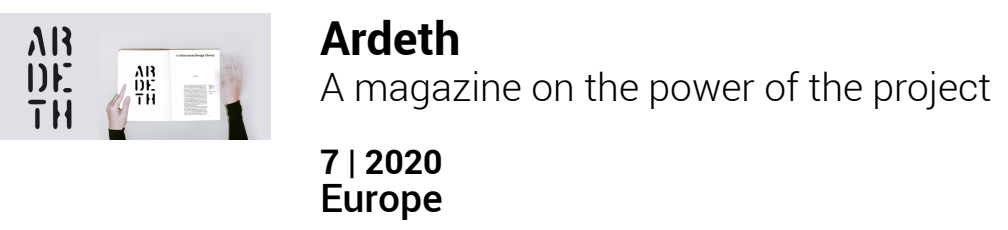

\title{
La riscrittura di un territorio
}

Le pratiche turistiche e la costruzione di un paesaggio abitato su un supporto naturale

The Rewriting of a Territory. Tourist Practices and the Construction of a

Domestic Model for an Inhabited Landscape

Silvana De Bari, Valerio Di Festa e Stefania Iraci Sareri

\section{(2) OpenEdition}

\section{Journals}

\section{Edizione digitale}

URL: https://journals.openedition.org/ardeth/1918

ISSN: 2611-934X

\section{Editore}

Rosenberg \& Sellier

\section{Edizione cartacea}

Data di pubblicazione: 1 décembre 2020

Paginazione: 143-155

ISSN: 2532-6457

\section{Notizia bibliografica digitale}

Silvana De Bari, Valerio Di Festa e Stefania Iraci Sareri, «La riscrittura di un territorio», Ardeth [Online], 7 | 2020, online dal 01 juin 2021, consultato il 25 juin 2021. URL: http://journals.openedition.org/ ardeth/1918 


\title{
La riscrittura di un territorio. Le pratiche turistiche e la costruzione di un paesaggio abitato su un supporto naturale
}

\author{
Silvana De Bari (1), Valerio Di Festa (2) \\ Stefania Iraci Sareri (3)
}

Abstract / The Rewriting of a Territory. Tourist Practices and the Construction of a Domestic Model for an Inhabited Landscape.

Temporary mobility phenomenons are changing the consistency of European geographies and are among the processes that rewrite our territory. Hence a reflection that tries, through a design vision, to investigate the role of nature: can it be considered as a rigid and complex support for those non-permanent housing practices that more than others rewrite and change the territory?

Are infrastructures only elements of a pseudo-sacral hierarchy or can they still be reinterpreted as tools for rewriting inhabited landscapes?

This rough and heterogeneous landscape becomes, within this conceptual frame, the support of all practices related to the sphere of living.
Affiliatio

(1) (2) (3) Ricercatore indipendente

Contacts:

(1) silvana [dot] debari [at] gmail [dot] com

(2) difestavalerio [at] gmail [dot] com

(3) stefaniairacisareri [at] gmail [dot] com

Received:

21 April 2020

Accepted:

16 December 2020

DOI:

10.17454/ARDETH07.09

ARDETH \#07 


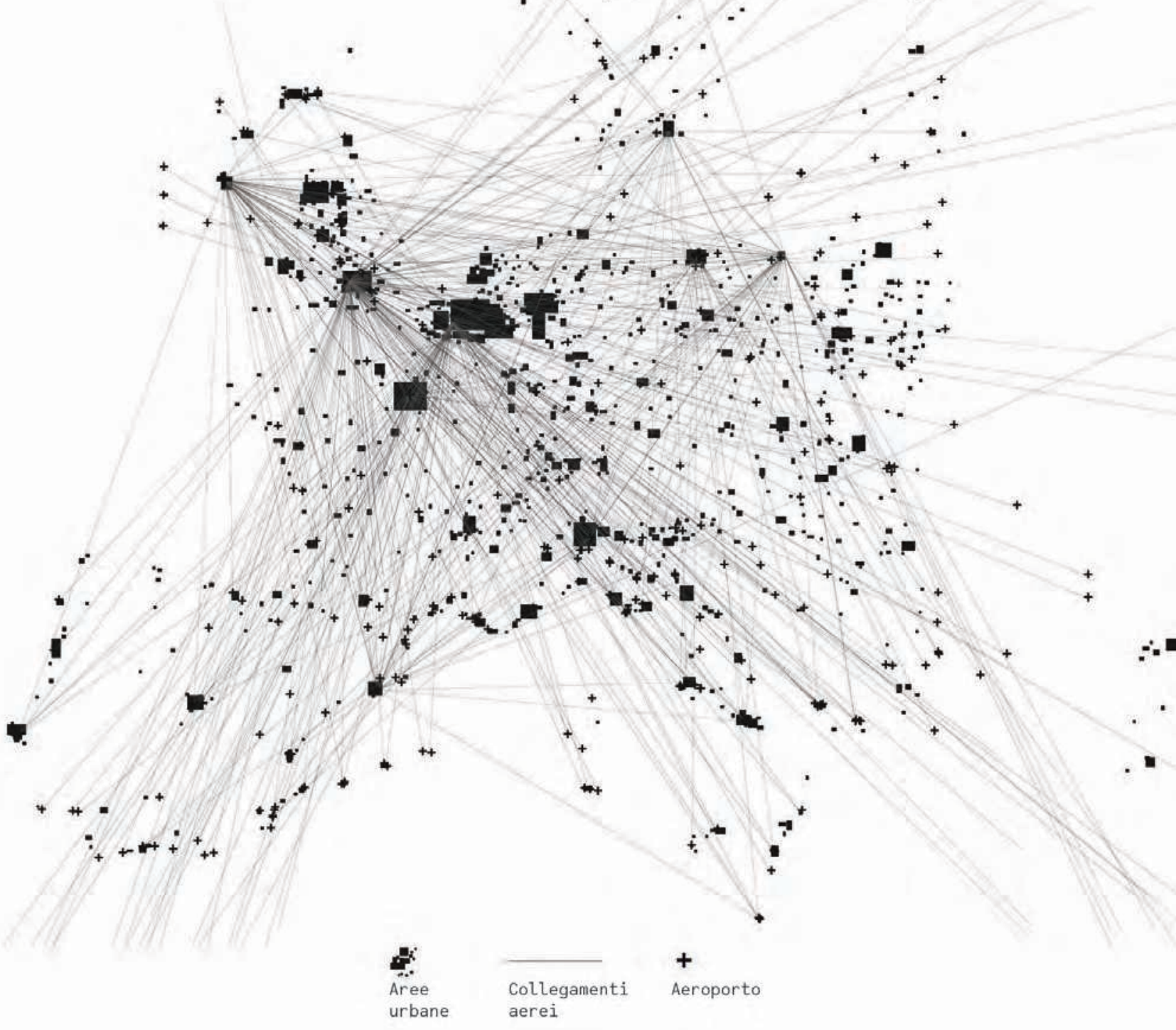

Fig. 1 - Low-cost

Europe Le infrastrutture - fisiche e dig tali - che supportano le pratiche turistiche hanno modificato il concetto di distanza: bus station e aeroporti in prossimità di grossi centri urbani incanalano flussi $\mathrm{m}$ gratori che circolano con biglietti low-cost. Queste connessioni definiscono nuove polarità e diverse prossimità geografiche in termini di distanza spaziale e temporale.

\section{Bordi, confini e migrazioni}

Se fosse possibile leggere in chiave positivista la moderna separazione tra i mondi naturali e quelli creati dall'uomo, il nostro mondo fatto da geografie rizomatiche, da reti ibride e da assemblaggi annidati potrebbe essere chiamato, come è stato suggerito da Neri Oxman (2016), "età dell'Entaglement”. La metafora quantistica dell'Entaglement è emblematica per descrivere le nuove geografie urbane e non-urbane che investono il territorio europeo. Uno spazio geografico sovrascritto dove tutti i confini sembrano sciogliersi e non esiste più corrispondenza polare tra ordine e disordine; dove l'attività umana diventa agente atmosferico e i paesaggi si trasformano in infrastruttura. L'Europa diventa il crocevia di reti globalizzate, hotspot di flussi di persone, capitali, dati e merci. Le migrazioni contemporanee di popolazioni non stanziali, di cui il turismo rappresenta l'espressione più deflagrante, riplasmano i territori, definiscono possibili ordini e confini, riscrivono nuove geografie. 


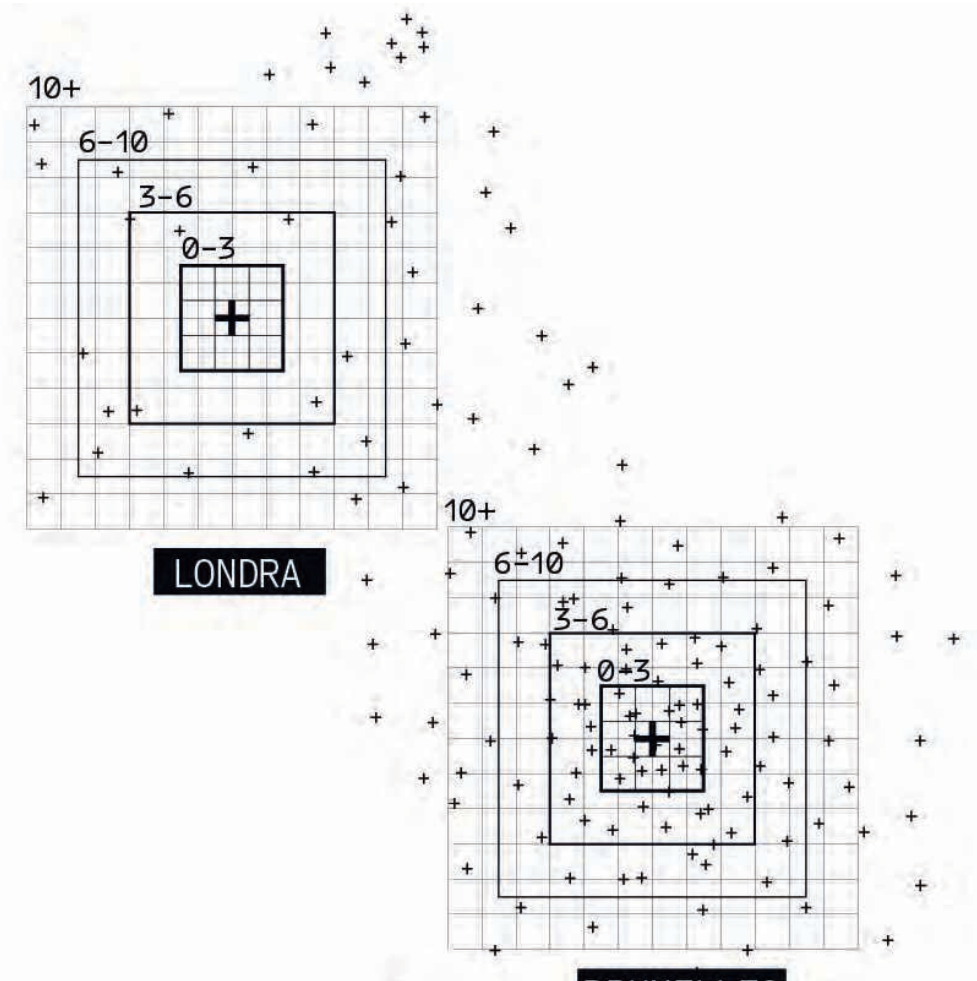

\section{BRUXELLES}

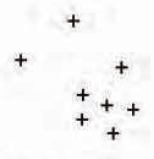

Città e luoghi di interese

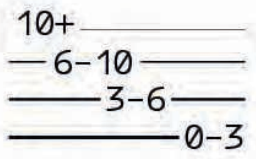

Ore di

percorrenza

in autobus

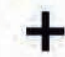

Aeroporto

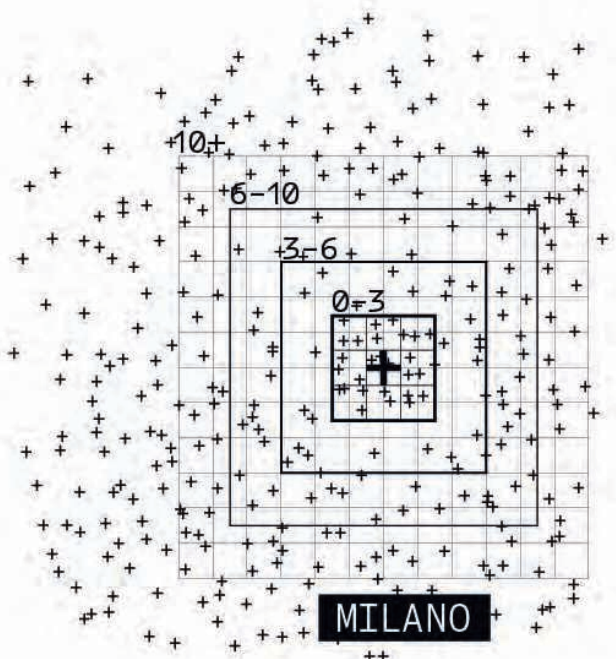


Fig. 2a - II nuovo concetto di distanza. I diagrammi analizzano il nuovo concetto di distanza rispetto alle variabili di tempo e movimento. I nuovi sistemi di infrastrutture fisiche hanno cambiato le caratteristiche geografiche dei luoghi. Londra e Bruxelles non sono più separate dal Canale della Manica ma da 8 ore di bus.

Fig. 2b - II nuovo concetto di distanza. La rete backdoor city, costruita dalle compagnie low-cost attraverso il posizionamento di piccoli aeroporti nelle vicinanze di grandi città, sta ampliando i raggi delle aree facilmente raggiungibili in prossimità di grossi centri urbani inglobando aree - urbane e rurali - di nuovo interesse turistico.

Fig. 3 - Le pratiche del turismo: flussi, insediamenti, aree di interesse.

I flussi turistici che investono i territori adriatici si confrontano con un territorio dalle differenti caratteristiche naturali che si relaziona con insediamenti urbani attrezzati in forme diverse per l'accoglienza turistica: gli sporadici insediamenti diffusi sulla costa balcanica si confrontano con l'edificato massivo che si srotola lungo la costa italiana, saturandola.

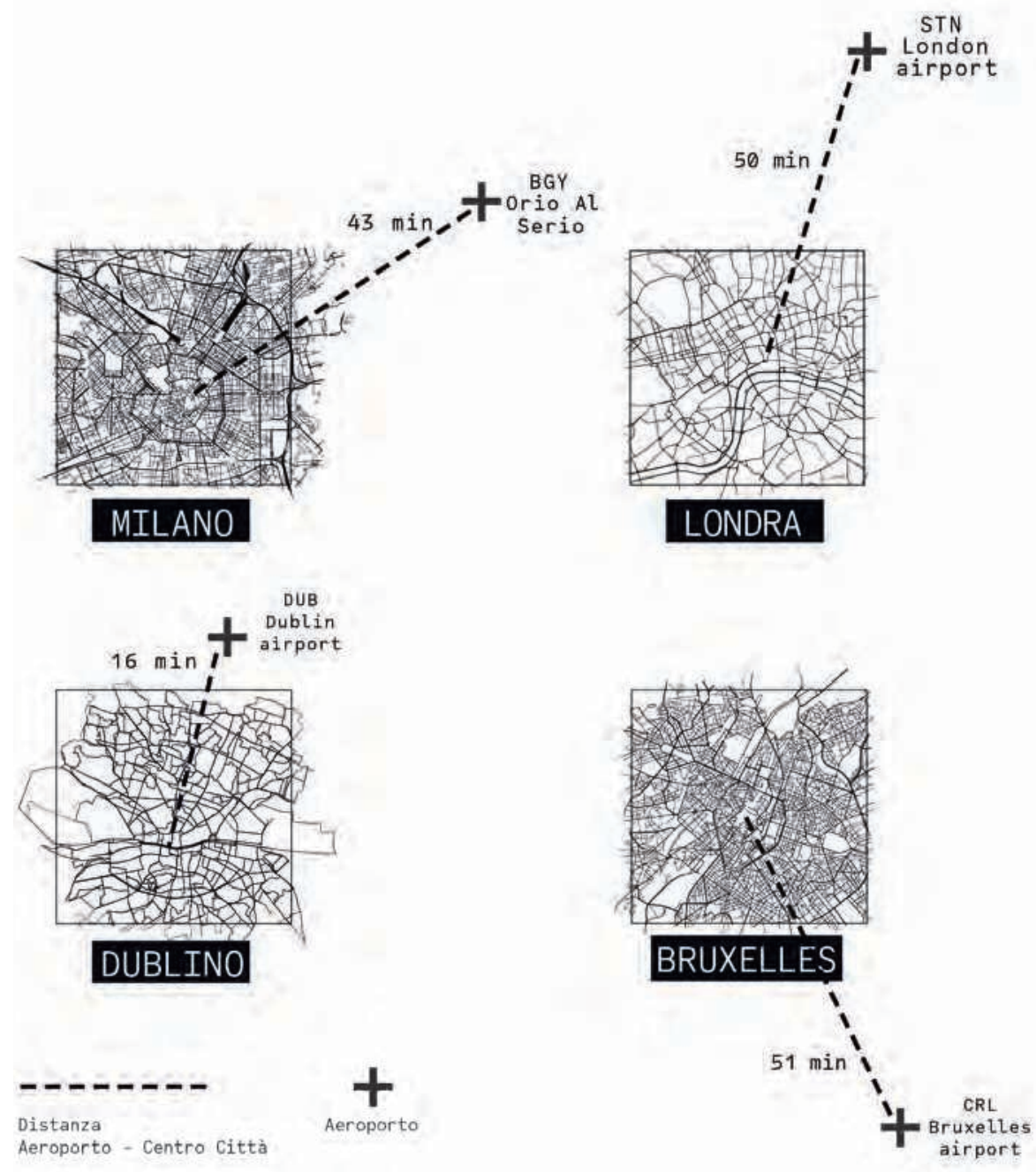

Il turismo, quindi, diventa il fenomeno spaziale per eccellenza, esso fa dell'osservazione del territorio la sua accezione più ampia (Di Campli, 2009). Una delle caratteristiche fondamentali del turismo moderno risiede nel bisogno di evasione da una condizione ordinaria per mezzo del viaggio. In funzione di questa necessità, questo fenomeno geografico costituisce dei veri e propri paesaggi turistici, scardinando i processi relazionali gerarchici tradizionali. In questo quadro è appropriato leggere il turismo attraverso uno scambio più chiaro e definito con le dotazioni naturali che contraddistinguono i luoghi. Queste naturalità evidenti e incontaminate; di riserve verdi, opache e luminose, rappresentano lo strato vibrante del territorio che, in quanto oggetto di interesse all'accoglienza turistica, è sincronico alle pratiche abitative temporanee. 


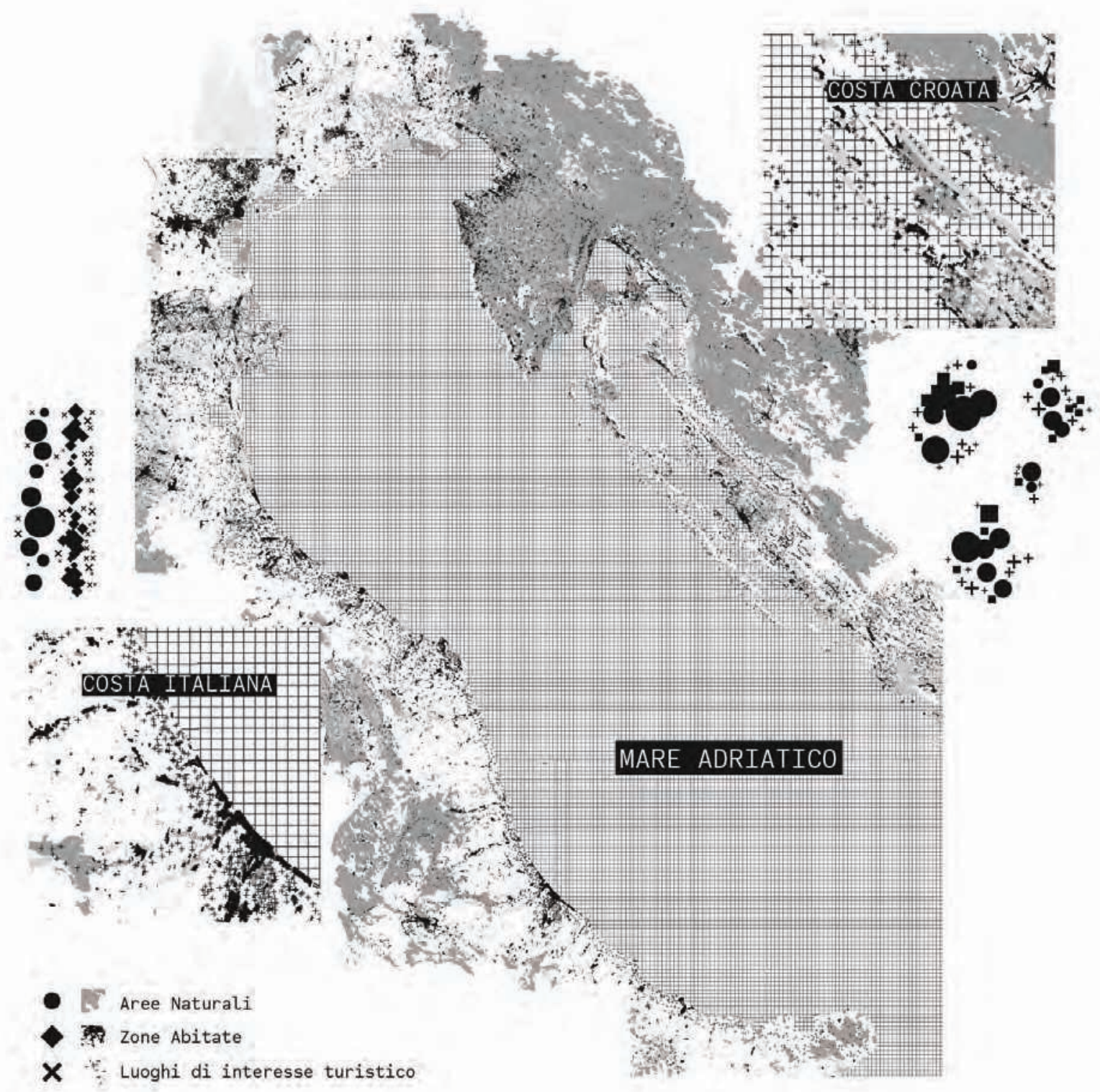

Nell'epoca dell'Antropocene, lo spazio turistico presenta numerose sfaccettature. Nella maggior parte dei casi, è un'enclave separata dal contesto circostante; è uno spazio centripeto, circoscritto, che si richiude su se stesso alla ricerca di una configurazione esaustiva del mondo. In altri, quest'ultimo tende a diventare non-omogeneo e segnato da pratiche in gran parte oscillanti. È quello che succede sulle coste del Mar Adriatico, milieu della ricerca progettuale presentata; qui l'esperienza turistica, nella sua accezione nomade e complessa, ha andamenti stagionali, percorribilità diverse e sovrapposizione varie di obiettivi e desideri. La costruzione di immaginari possibili e l'avvio di un processo progettuale può essere lo strumento che permette un'estrazione di nuovi valori e la valorizzazione di caratteristiche intrinseche presenti nei paesaggi (turistici) abitati. 


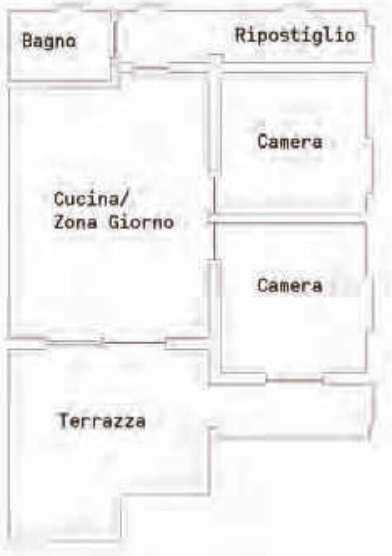

Abitazione per residenti

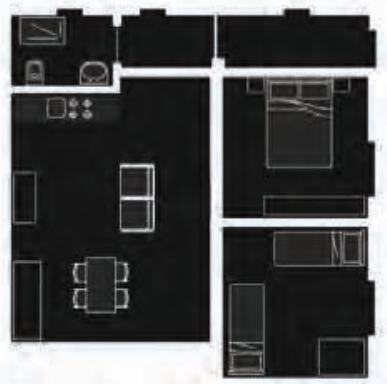

Arredi

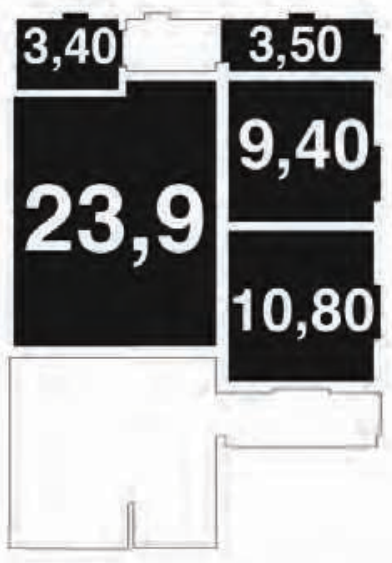

Dimensione spazi minimi

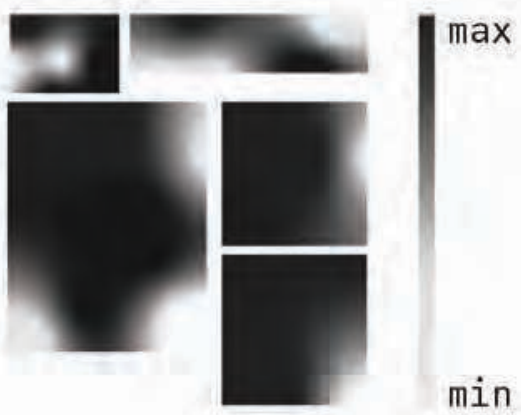

Utilizzo degli spazi domestici
Fig. 4a - II ridimensionamento dello spazio domestico Il confronto analitico di diversi alloggi residenziali sulla costa balcanica, fatto sulla base di una ricerca diretta, ha portato alla definizione degli spaz e degli ambienti della residenza che vengono maggiormente utilizzati da parte dei residenti, considerando come questi vivono l'alloggio in modo dilatato nel tempo.

\section{Il paesaggio abitato}

Leggere le infrastrutture attraverso una lente multiforme e transcalare ci sollecita a porre domande variegate e multidirezionali e a discostarci da una possibile lettura puramente funzionalista (Ballestero, 2016). Questa calibrazione ha la potenza per produrre nuovi scenari possibili, nuove figurazioni spaziali e nuove letture infrastrutturali capaci di riscrivere i particolarismi di infiniti territori. Territori in cui il naturale e il sociale si amalgamano e costituiscono una nuova entità. Nei bordi della "pianura liquida” di Fernand Braudel, natura e artificio condividono lo stesso piano, acquisiscono un ruolo preminente dove il loro potere spaziale affiora da diverse e mutevoli disposizioni di materiali, corpi, idee e agenti; è una trans-mutazione del paesaggio dove la natura non viene sostituita dall'ambiente costruito, ma dove quest'ultimo dovrà piegarsi a essa. (Helmirich, 2014). 


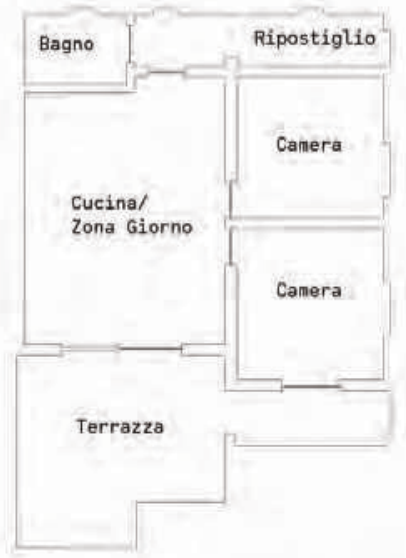

Abitazione per turista

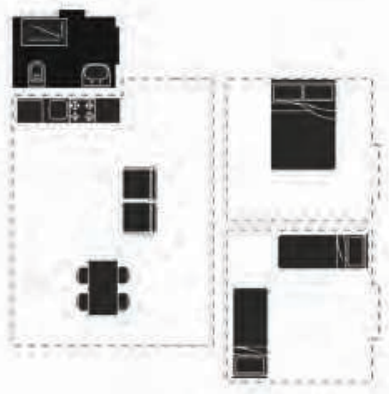

Arredi

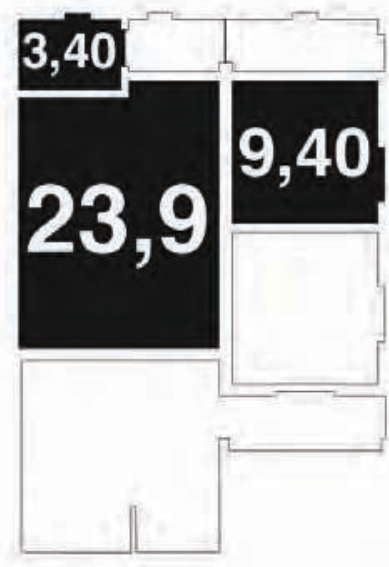

Dimensione spazi minimi

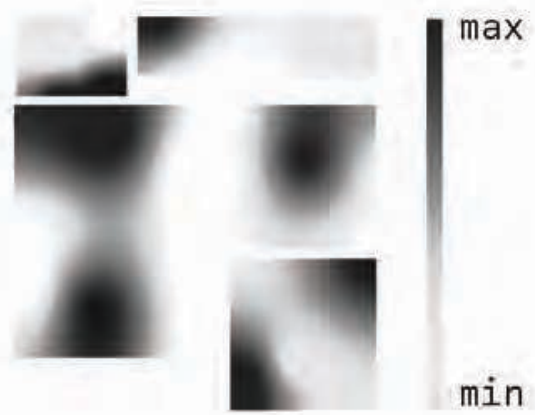

Utilizzo degli spazi domestici
La netta alternanza tra gli elementi urbani e le grandi placche naturali permette di percepire la natura come vuoto, sfondo della scena. Provando a invertire la gerarchia valoriale tra ciò che è pieno e ciò che è vuoto, la natura diventa materia di progetto e non più residuo e luogo di assen$\mathrm{za}$, assume un valore architettonico, spazialmente determinato (Russi 2019). Ma allora in che modo il territorio e la natura possono accogliere pratiche temporanee dell'abitare?

Il fenomeno turistico incide su questo sistema attraverso la sua capacità di riscrittura. Vivere e fare esperienza di un territorio, nei suoi intrecci, nei suoi differenti tasselli, nei suoi spazi più confinati e descrittivi vuol dire modificarlo costruendo al tempo stesso un nuovo paesaggio: il paesaggio abitato. Il paesaggio abitato è un sistema di corpi, di relazioni con lo spazio quasi fenomenologiche, di conflitti e prossimità. Uno spazio che vissuto attraverso l'ingombro dei corpi (Bianchetti, 2020), acquisisce
Fig. 4b - II ridimensionamento dello spazio domestico L'analisi sull'uso dello spazio dell'alloggio turistico tipico, spesso copia dell'alloggio dei residenti, ha evidenziato un sovradimensionamento e la overdotazione di spazi. Chi vive l'alloggio in modo temporaneo in questi luoghi necessita di pochi spazi costruiti, legati perlopiù a funzioni quotidiane specifiche 
Fig. 4c - II ridimensionamento dello spazio domestico. Definire lo spazio domestico per turista significa quindi svestirlo di quegli ambienti poco utilizzati nel quadro delle esigenze di utilizzatori non stanziali. Lo svelamento dei soli ambienti della casa che necessitano di elementi di consistenza fisica diviene punto di partenza per ridefinire gli spazi domestici - aperti e costruiti - dell'alloggio turistico.

Fig. 4d - II ridimensionamento dello spazio domestico. Le tre soluzioni progettuali sono unità minime che rispondono alle esigenze del turismo romantico contemporaneo, mosso dal desiderio di un territorio esotico da vivere temporaneamente, diverso dagli spazi del quotidiano. Queste configurazi ni spaziali invitano il turista a considerare lo spazio esterno come ambiente domestico, suggerendone un possibile utilizzo.

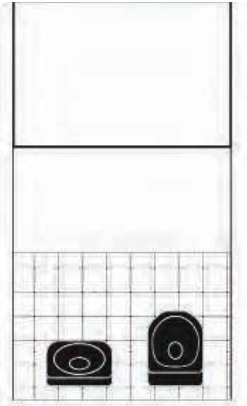

\section{Servizi}

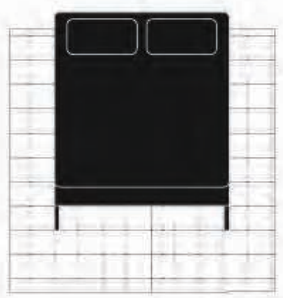

\section{Letto}

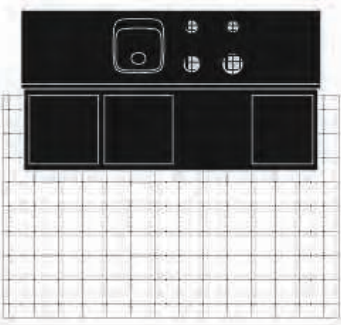

\section{Cucina}

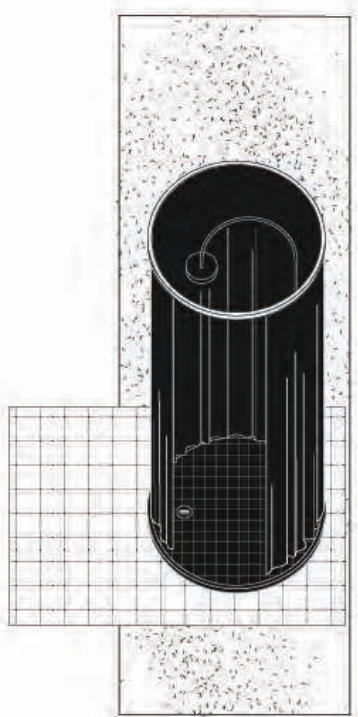

Doccia 

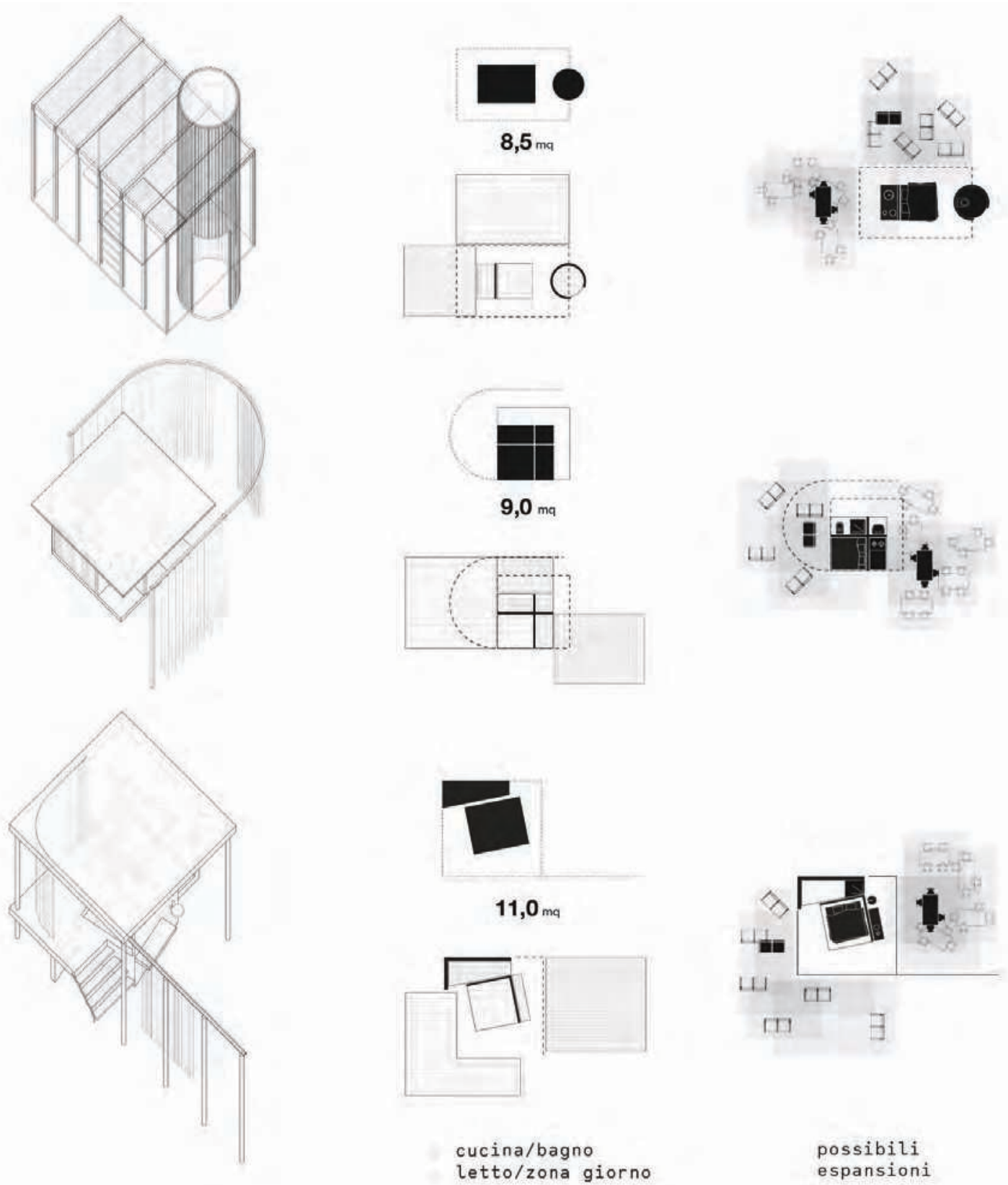

uno spessore più ampio, diventa volume. Il modello abitativo turistico necessità di un processo di minorazione e di asciugatura dello spazio domestico; di un resize the oversize della casa vacanze. La casa è diventata uno spazio chiuso, un contenitore introverso che lascia al di fuori la natura. Uno spazio in cui l'architettura rimane sullo sfondo adornata da una serie di oggetti e suppellettili inutili alle pratiche dell'abitare temporaneo. La natura diventa supporto delle pratiche collettive del turismo. 


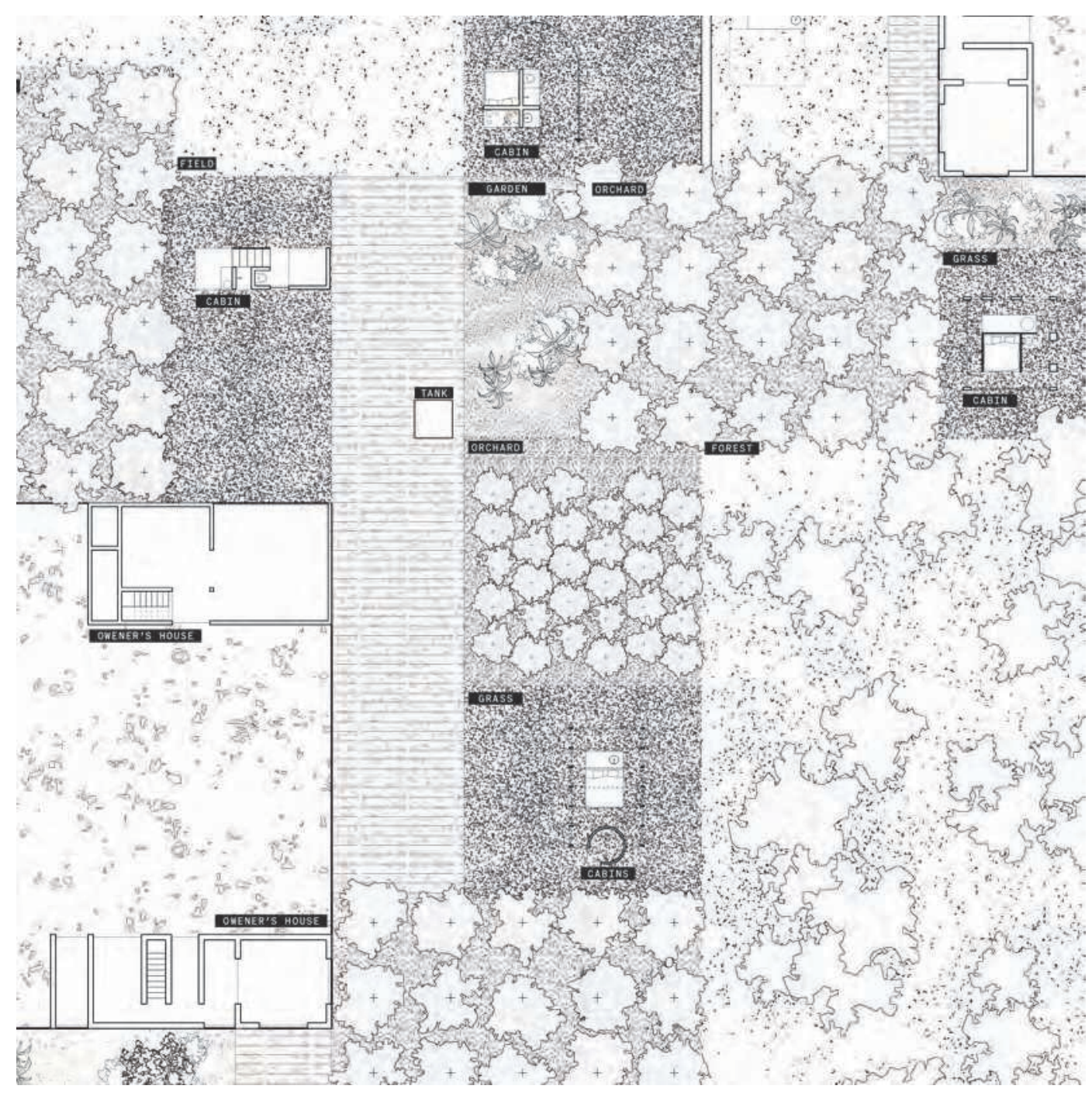

Lo schema compositivo proposto ipotizza l'inversione dei ruoli formali e strutturali di figura e suolo e ridefinisce la relazione tra l'uomo e il paesaggio. Lo spazio domestico viene disvelato, ridotto al solo spazio necessariamente privato. Unità minime, boschi, radure, spazi soglia, tasselli vegetali, reti energetico-idriche o di raccolta dell'acqua piovana sono tutti elementi di questa grammatica, che alla scala della costituzione della regola risulta fortemente isotropa. È, in realtà, un sistema formato da una varietà di elementi gerarchici che si accostano, si sovrappongono, si sottraggono l'un l'altro e si alternano nelle forme e nelle composizioni, in uno spazio in cui è continuamente compresso o dilatato l'equilibrio tra corpo, soggetti e sfondo.

La dimensione spaziale della prossimità e del conflitto è sovrascritta dall'istantaneità delle pratiche individuali e collettive e dai loro imprevisti. Dalle riflessioni espresse, le risposte progettuali in ambito turistico de- 


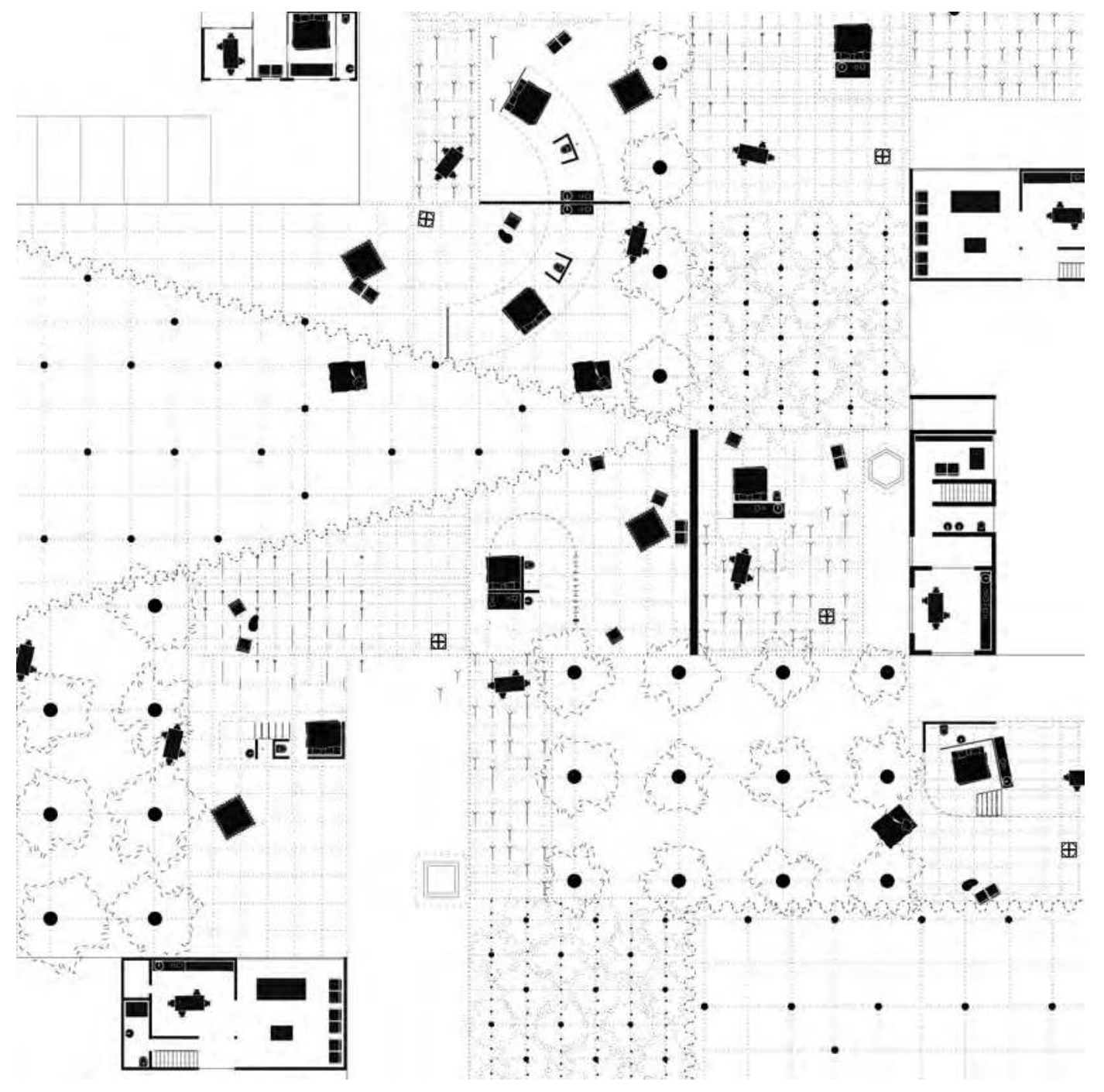

Fig. 6 - Abitare il

territorio

Lo spazio esterno

assume un'impor-

tanza centrale nella

configurazione degli

spazi di accoglienza

turistica, in cui l'uomo

è soggetto in cerca di

una condizione di ar-

monia con il territorio.

II rapporto edonistico

tra uomo e natura si

realizza pienamente

quando il paesaggio

si fa ambiente in cui

l'uomo è immerso. 


\section{Bibliografia}

Albert, M., Jacobson, D. (eds), Identities, Borders, Orders: Rethinking Internationl Relation Theory, Minneapolis, University of Minnesota Press.

Akawi, N. (2018), Mapping Borderlands, in M. Oter Verzier, N. Axel, Work, Body, Leisure, Rotterdam, Het Nieuwe Institut.

Appadurai, A. (1996), Modernity at Large. Cultural Dimensions of Globalization, Minneapolis, University of Minnesota.

Appadurai, A. (2000), Grassroots Globalization and the Research Imagination in 'Public Culture', Durham, Duke University Press.

Ballestero, A. (2016), Living with Aquifers, "E-Flux Architecture+Liquid" [Online]. Available at: https://www.e-flux.com/architecture/liquid-utility/259651/living-with-aquifers/ [Accessed: 18 aprile 2020].

Bianchetti, C. (2016), Spazi che contano. Il progetto urbanistico in epoca neo-liberale, Roma, Donzelli.

Bianchetti, C. (2020), Corpi tra spazio e progetto, Milano, Mimesis.

Branzi, A. (2006), Modernità debole e diffusa. Il mondo del progetto all'inizio del XXI secolo, Milano, Skira.

Braudel, F. (1986), Civiltà e imperi del Mediterraneo nell'età di Filippo II, vol. I, Torino, Einaudi.

Castells, M. (1989), The informational city, Oxford, Blackwell.

Di Campli, A. (2009), Adriatico dopo la crisi, Trento, List LAB.

Gissen, D. (2009), Subnature. Architecture's Other Environments, New-York, Princeton Architectural Press.

Harvey, D. (1989), The Condition of Postmodernity: An Enquiry into the Origins of Cultural Change, Oxford, Blackwell.

Helmrich, S. (2014), how to Hide a Island, "New Geographies”, n. 6, Harvard Graduate School.

Indovina, F. (2019), Nuove forme di valorizzazione del Capitale. Dalla città del Capitale alla città del Consumo, in C. Bianchetti (ed.), Territorio e produzione, Macerata, Quodlibet.

Koolhas, R., Mau, B. (eds) (1995), S, M, L, XL, New York, The Monacelli Press.

Lyste, C. (2016), Learning from Logistic. How network change the city, Basilea, Birkhauser.

Oxman, N. (2016), Age of Entaglement, "Journal of Design an Science”, dati bibliografici, n. 1.

Quaini, M. (2006), L'ombra del paesaggio, Parma, Diabasis.

Roy, A. (2009), The $21^{\text {st }}$ Century Metropolis: New Geographies of Theory, "Regional Studies”, vol. 43, n. 6.

Russi, N. (2019), Background. Il progetto del vuoto, Macerata, Quodlibet.

Steyerl, H. (2016), A Sea of Data: Apophenia and Pattern (Mis-)Recognition "E-Flux Architecture”, n. 7 [Online]. Available at: https://www.e-flux.com/journal/72/60480/ a-sea-of-data-apophenia-and-pattern-mis-recognition/ [Accessed: 18 aprile 2020].

Strang, G. (1997), Infrastructure as landscape, “Places”, n. 10. 